\title{
Predictors of Epstein-Barr virus serostatus in young people in England
}

Joanne R. Winter ${ }^{1 *}$ (D) Graham S. Taylor ${ }^{2 \dagger}$, Olivia G. Thomas², Charlotte Jackson ${ }^{1,3}$, Joanna E. A. Lewis ${ }^{4 \dagger}$ and

Helen R. Stagg ${ }^{1,5^{*}}$

\begin{abstract}
Background: Epstein-Barr virus (EBV) is an important human pathogen which causes lifelong infection of $>90 \%$ people globally and is linked to infectious mononucleosis (arising from infection in the later teenage years) and several types of cancer. Vaccines against EBV are in development. In order to determine the most cost-effective public health strategy for vaccine deployment, setting-specific data on the age at EBV acquisition and risk factors for early infection are required. Such data are also important to inform mathematical models of EBV transmission that can determine the required target product profile of vaccine characteristics. We thus aimed to examine risk factors for EBV infection in young people in England, in order to improve our understanding of EBV epidemiology and guide future vaccination strategies.

Methods: The Health Survey for England (HSE) is an annual, cross-sectional representative survey of households in England during which data are collected via questionnaires and blood samples. We randomly selected individuals who participated in the HSE 2002, aiming for 25 participants of each sex in each single year age group from 11 to 24 years. Stored samples were tested for EBV and cytomegalovirus (CMV) antibodies. We undertook descriptive and regression analyses of EBV seroprevalence and risk factors for infection.
\end{abstract}

Results: Demographic data and serostatus were available for 732 individuals. EBV seroprevalence was strongly associated with age, increasing from $60.4 \%$ in 11-14 year olds throughout adolescence (68.6\% in 15-18 year olds) and stabilising by early adulthood (93.0\% in those aged 22-24 years). In univariable and multivariable logistic regression models, ethnicity was associated with serostatus (adjusted odds ratio for seropositivity among individuals of other ethnicity versus white individuals 2.33 [95\% confidence interval 1.13-4.78]). Smoking was less strongly associated with EBV seropositivity.

Conclusions: By the age of 11 years, EBV infection is present in over half the population, although age is not the only factor associated with serostatus. Knowledge of the distribution of infection in the UK population is critical for determining future vaccination policies, e.g. comparing general versus selectively targeted vaccination strategies.

Keywords: Epstein-Barr virus, Serostatus, Infectious mononucleosis, Cancer, Transmission, Risk factors

\section{Background}

Epstein-Barr Virus (EBV) is a herpesvirus that infects $90-95 \%$ of humans, causing lifelong infection $[1,2]$. EBV infection during childhood is generally asymptomatic, however acquisition of EBV during adolescence or early adulthood often causes infectious mononucleosis (IM), [3] which can cause substantial morbidity during

\footnotetext{
* Correspondence: helen.stagg@ed.ac.uk

† Graham S. Taylor and Joanna E. A. Lewis contributed equally to this work.

${ }^{1}$ Centre for Molecular Epidemiology and Translational Research, Institute for Global Health, University College London, London, UK

Full list of author information is available at the end of the article
}

important educational periods in adolescents and young adults $[4,5]$. EBV is associated with $1 \%$ of global cancers, particularly Hodgkin's lymphoma, Burkitt's lymphoma, nasopharyngeal cancer and gastric cancer [6].

EBV infection is not currently treatable nor preventable by vaccination; however, vaccine candidates are in development. In phase II trials, a first-generation vaccine administered to healthy seronegative volunteers aged 16-25 years demonstrated protection against IM but not EBV infection [7]. Second-generation vaccines elicited higher levels of antibody responses in animal models, [8]

(c) The Author(s). 2019 Open Access This article is distributed under the terms of the Creative Commons Attribution 4.0 International License (http://creativecommons.org/licenses/by/4.0/), which permits unrestricted use, distribution, and 
and first-in-human trials are likely to begin soon. Mathematical modelling of different vaccination strategies is essential to determine the effectiveness and costeffectiveness of different vaccination strategies for reducing rates of EBV infection, IM, and EBV-associated cancers, taking into account factors such as vaccine efficacy, duration of protection and differing outcomes according to age at infection.

A greater understanding of EBV epidemiology, including the dynamics of EBV infection in different subpopulations, is necessary for the development of such models. EBV seroprevalence increases with age; $90-95 \%$ of people globally are infected by age 25 , whilst $5-10 \%$ remain seronegative throughout life [9]. The best public health strategy for the deployment of an infectionpreventing vaccine may vary between settings; infection appears to occur at younger ages in resource-limited countries and thus children will need to be vaccinated early [10-12]. However, if the duration of vaccineinduced protection is not lengthy, vaccinated individuals may become susceptible to natural infection at an age where the consequences of infection are more severe, for example leading to IM or cancer [13].

Additionally, sub-optimal vaccine coverage even of a vaccine with a long duration of protection will lead to a higher age at infection amongst those who remain unvaccinated. In such situations it may be better to delay vaccination until the pre-teenage years, targeting individuals who remain EBV seronegative. Alternatively, a vaccine protecting against IM and EBV-associated diseases (such as certain cancers) could be administered to older children as they approach adolescence, which may be effective even with a shorter duration of protection. After the licensing of vaccine candidates, strategic discussions will need to take place nationally and be informed by accurate national data on the epidemiology of EBV infection.

In the United Kingdom, EBV seroprevalence increases rapidly in very young children, reaching 21 and $51 \%$ by the age of two years in children of white and Pakistani ethnicity, respectively [14]. Another study showed that EBV seroprevalence then remained relatively constant, at around 55\%, between the ages of five and 11 years [15]. EBV seroprevalence was estimated at $75 \%$ in university students at 19 years and $92 \%$ by the age of 22 years [16]. We recently published summary data on the seroprevalence of EBV in adolescents in England [13]; however, to date no study has investigated factors associated with seropositivity that could inform a targeted vaccination strategy.

Our aim was to investigate the sociodemographic and lifestyle factors, particularly age, associated with EBV serostatus in children and young adults in England, and to discuss the implications of our findings for future EBV vaccination policy.

\section{Methods}

\section{Study population}

The Health Survey for England (HSE) is an annual, cross-sectional, representative survey of households in England. Its methods are described in detail elsewhere [17]. For this study, and in order to parameterise a model of EBV transmission, [13] we randomly selected individuals who participated in the 2002 HSE; 2002 was the most recent year in which survey participants gave consent for future studies to test their blood samples for blood-borne viruses. Our aim was to include 25 participants of each sex in each single year age group from 11 to 24 years, in order to fill a gap in the literature and capture the years at which infection is most likely to have clinical consequences. The participant IDs were selected randomly by the HSE, however it was not possible at the time of sampling to determine whether the samples had already been used. As a result, more than 25 IDs were selected for each age-sex group to ensure there were sufficient samples for our analysis, and therefore there are not exactly 25 samples in each group (Additional file 1: Table S1).

\section{Measuring seroprevalence of Epstein-Barr virus and cytomegalovirus infection}

Stored blood serum samples collected between January 2002 and March 2003 were obtained from the HSE. Samples were posted to the laboratory within two days, where they were centrifuged, and the remaining serum was frozen and stored at $-40^{\circ} \mathrm{C}$ until they were analysed, which was completed in September 2017 [18].

EBV virus capsid antigen (VCA)-specific IgG and $\mathrm{CMV}$-specific IgG were detected in serum samples using commercial ELISA kits obtained from EUROIMMUN, Germany (EI2791-9601-G, EI2570-9601G). Assays were performed according to manufacturer's instructions and serum antibody concentrations were calculated using a standard curve. Data on the performance of the assays are detailed in Additional file 1: Table S2. Results were presented in relative units $(\mathrm{RU} / \mathrm{mL}) ;<16 \mathrm{RU} / \mathrm{mL}$ samples were considered negative, $\leq 16$ to $<22 \mathrm{RU} / \mathrm{mL}$ borderline and $\geq 22 \mathrm{RU} / \mathrm{mL}$ positive. Borderline results from the EBV VCA IgG ELISA were subsequently subjected to reanalysis with an EBV immunoblot assay (EUROIMMUN, Germany, DY2790G) which revealed all borderline serum samples $(n=5)$ had reactivity to alternative EBV antigens; they were therefore considered seropositive.

\section{Statistical analysis}

Data were analysed in Stata version 15.0. We weighted our sample, using the svy commands in Stata, to be representative of the English population in 2002 with respect to age and sex, utilising data from the Office for National Statistics [19]. All stated percentages are 
weighted. Descriptive analyses of the study population were undertaken. ArcMap 10.3.1 was used to create a map of EBV seroprevalence by English Government Office Region [20].

To investigate factors associated with being seropositive for EBV, we undertook logistic regression modelling. A causal inference framework was used to determine $a$ priori factors to be included in multivariable models, from the available data collected in the HSE. We built two multivariable regression models.

A 'whole-population' model, which included our entire study population, examined the following factors: age, sex, ethnicity (categorised as 'white' or 'other' due to small numbers of non-white participants), body mass index (BMI; categorised as 'underweight' [BMI <20], 'healthy weight' $[20-<25]$, 'overweight' $[25-<30]$ or 'obese' [ $\geq 30]$ ), region of England and CMV serostatus.

A second 'adults-only' model was restricted to individuals aged $\geq 16$ years, and additionally included factors for which data was only available for adults; smoking status (never smoked, current smoker, smoked in past) and occupational category from the National Statistics Socioeconomic classification (NS-SEC) [21]. The NS-SEC categorises occupations into higher managerial and professional roles (involving strategy/supervision), intermediate occupations (typically clerical, sales, service or technical positions which do not involve general planning or supervision), routine and manual occupations (involving basic labour), never worked or long-term unemployed, and other. We excluded individuals missing data on one or more variables.

Planned sensitivity analyses investigated the impact of excluding CMV serostatus as a predictor of EBV serostatus, and the impact of classifying the originally indeterminate serological results as seronegative rather than seropositive.

\section{Ethical approval}

This study was approved by the University College London Research Ethics Committee (5683/002). The HSE obtained informed written consent for blood samples to be collected and stored for future analyses [17].

\section{Results}

Our study sample included 732 individuals aged 11-24 years, of whom 547 (74.6\%) were EBV-seropositive. The characteristics of seropositive individuals are shown in Table 1.

EBV serostatus was associated with CMV serostatus; $72.6 \%$ of CMV-seronegative individuals were EBV seropositive compared to $80.9 \% \mathrm{CMV}$-seropositive individuals $\left(\chi^{2}\right.$ test $P=0.04$, Table 1$)$. Considerable variation in EBV seroprevalence was observed by UK region (Fig. 1, Table 1). EBV seropositivity increased with age, from $39.6 \%$ at $11-$ 14 years to $93.0 \%$ at $22-24$ years (Fig. 2).
Factors associated with EBV seropositivity were largely consistent between the univariable and multivariable models (Table 2). Increasing age was associated with increased EBV seroprevalence (adjusted odds ratio [aOR] 9.16 [95\% confidence interval (CI) 4.38-19.14] for people aged 22-24 years compared to those aged 11-14 years), as was non-white ethnicity (aOR 2.33 [1.134.78]). CMV seropositivity was associated with EBV seropositivity in the 'adults-only' multivariable model (aOR 2.16 [1.05-4.43]) but not in the 'whole population' model (aOR 1.25 [0.79-1.98]).

Among adults, EBV seropositivity was higher among those who currently smoked (aOR 4.29 [2.13-8.65]), than those who had never smoked. There was no evidence of associations between sex, BMI or occupational category and EBV serostatus.

In sensitivity analyses, we firstly excluded CMV serostatus as a predictor of EBV serostatus, and secondly we classed indeterminate serology results $(n=5)$ as seronegative rather than seropositive. Both sensitivity analyses showed results consistent with our main analyses (Additional file 1: Table S3, Table S4).

\section{Discussion}

The importance of EBV as a cancer-causing pathogen has generated international interest in developing an anti-infection vaccine [22]. The cost-effectiveness of different strategies to deploy such vaccines will vary from setting to setting and is dependent on the epidemiology of the infection. For example, EBV's association with IM means that vaccines that do not produce lifelong immunity may be better targeted towards subgroups which are likely to acquire infection in adolescence. In this observational study of factors associated with EBV seroprevalence among young people in England in 2002, we explored the distribution of seroprevalence by age and the sources of additional variability. We found a substantial increase in EBV seroprevalence with age among our sample population, associations with ethnicity and smoking, and a potential association with CMV seroprevalence.

A series of studies have demonstrated that EBV is generally acquired pre-adulthood, and that this varies between settings [12]. Our findings regarding smoking fit with the prevailing narrative that there is an association between EBV and socioeconomic status, rather than smoking being an independent risk factor [12]. Unfortunately, we did not have a good measure of socioeconomic status in our analysis; the NS-SEC does not account for familial socioeconomic status during childhood, which is probably more relevant to EBV seroprevalence than individual occupational status in young adults, and we were unable to measure socioeconomic status in children at all. 
Table 1 The number and weighted percentage of individuals seropositive for EBV in England in 2002

\begin{tabular}{|c|c|c|c|c|}
\hline & \multicolumn{2}{|l|}{ Whole cohort } & \multicolumn{2}{|l|}{ Adults only } \\
\hline & & EBV seropositive & & EBV seropositive \\
\hline Variable & Total number & Number (weighted \%) & Total number & Number (weighted \%) \\
\hline Total & 732 & $547(74.6)$ & 472 & $389(74.6)$ \\
\hline \multicolumn{5}{|l|}{ Sex } \\
\hline Men & 364 & $266(72.5)$ & 234 & $185(78.8)$ \\
\hline Women & 368 & $281(76.6)$ & 238 & $204(86.0)$ \\
\hline \multicolumn{5}{|l|}{ CMV serostatus } \\
\hline CMV-seronegative & 557 & $406(72.6)$ & 346 & $275(79.6)$ \\
\hline CMV-seropositive & 175 & $141(80.9)$ & 126 & $114(90.5)$ \\
\hline \multicolumn{5}{|l|}{ Age last birthday } \\
\hline $11-14$ years & 208 & $125(60.4)$ & & \\
\hline $15-18$ years $\dagger$ & 212 & $145(68.6)$ & 160 & $112(70.1)$ \\
\hline $19-21$ years & 156 & $132(84.6)$ & 156 & $132(84.6)$ \\
\hline 22-24 years & 156 & $145(93.0)$ & 156 & $145(93.0)$ \\
\hline \multicolumn{5}{|l|}{ Ethnic group } \\
\hline White & 655 & $482(73.4)$ & 425 & $345(81.2)$ \\
\hline Other & 77 & $65(84.2)$ & 47 & $44(93.6)$ \\
\hline \multicolumn{5}{|l|}{ BMI } \\
\hline Healthy weight & 418 & $300(71.6)$ & 259 & $207(79.8)$ \\
\hline Underweight & 60 & $47(78.7)$ & 60 & $47(78.7)$ \\
\hline Overweight & 141 & $113(79.6)$ & 102 & $90(88.3)$ \\
\hline Obese & 87 & $65(74.8)$ & 35 & $31(89.1)$ \\
\hline Missing & 26 & $22(84.6)$ & 16 & $14(87.5)$ \\
\hline \multicolumn{5}{|l|}{ Region } \\
\hline East of England & 78 & $50(64.6)$ & 52 & $39(75.6)$ \\
\hline North East & 34 & $27(79.8)$ & 25 & $23(91.8)$ \\
\hline North West & 130 & $98(74.4)$ & 80 & $66(82.0)$ \\
\hline Yorkshire and The Humber & 82 & $70(85.0)$ & 58 & $51(87.9)$ \\
\hline East Midlands & 74 & $57(77.8)$ & 48 & $39(81.0)$ \\
\hline West Midlands & 70 & $46(65.3)$ & 33 & $24(73.0)$ \\
\hline London & 63 & $50(78.6)$ & 42 & $37(88.2)$ \\
\hline South East & 119 & $88(73.4)$ & 82 & $64(78.2)$ \\
\hline South West & 82 & $61(75.9)$ & 52 & $46(88.9)$ \\
\hline \multicolumn{5}{|l|}{ Ever smoked ${ }^{a}$} \\
\hline Never smoked & - & - & 186 & $137(73.8)$ \\
\hline Currently smoke & - & - & 134 & $124(92.5)$ \\
\hline Smoked in past & - & - & 147 & $124(84.4)$ \\
\hline Missing & - & - & 5 & $4(80.0)$ \\
\hline \multicolumn{5}{|l|}{ Occupational category ${ }^{\mathrm{a}}$} \\
\hline Higher managerial and professional & - & - & 83 & $71(85.9)$ \\
\hline Intermediate occupations & - & - & 69 & $61(88.3)$ \\
\hline Routine and manual occupations & - & - & 254 & $202(79.6)$ \\
\hline Never worked or long-term unemployed & - & - & 11 & $10(91.2)$ \\
\hline Other & - & - & 55 & $45(81.4)$ \\
\hline
\end{tabular}

${ }^{a}$ Adults aged $\geq 16$ years only $(n=472)$. $+16-18$ years for 'adult-only' model. Percentages account for the weighting of the sample to be representative of the English population in 2002 with respect to age and sex. BMI: body mass index, Cl confidence interval, CMV cytomegalovirus, EBV Epstein-Barr virus 


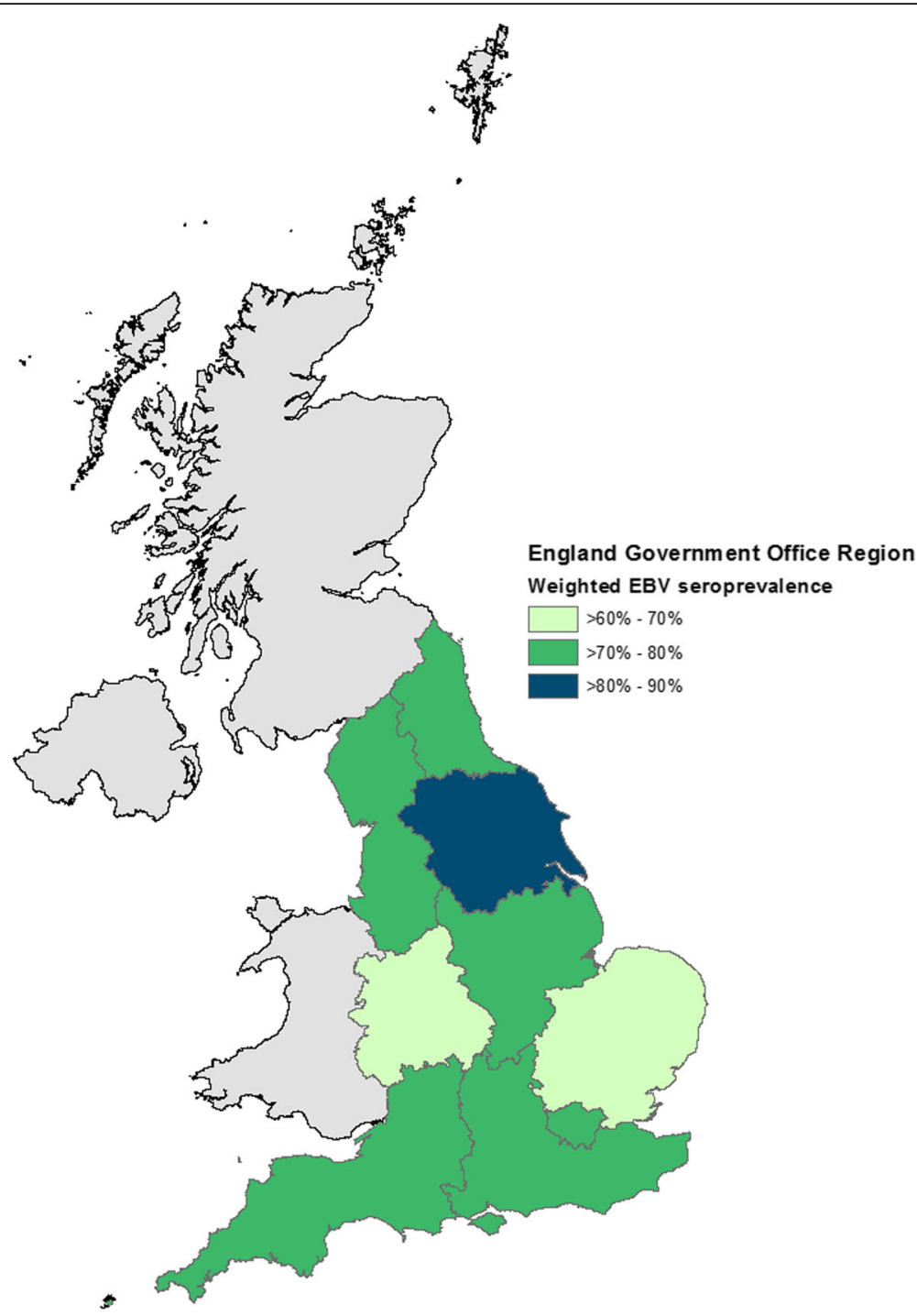

Fig. 1 Weighted Epstein-Barr virus seroprevalence by English Government Office Region in 2002. Contains National Statistics data@ Crown copyright and database right [2011] Contains public sector information licensed under the Open Government Licence v3.0

We found that EBV prevalence varied substantially between regions of the UK in univariable analyses and in the whole-cohort model, but not in the adults-only model, suggesting confounding between region and socioeconomic status. There was also a strong association between EBV seropositivity and ethnicities other than white, in both univariable and multivariable models. This may be the result of different mixing patterns (as people of ethnic minorities are more likely to live in larger households), different feeding practices, or residual confounding of socioeconomic status. CMV is another herpesvirus which infects a high proportion of the population from a young age, [23] and has also been associated with EBV in other settings [24, 25].

In England, EBV infects 55\% of the population by the age of 12 [15]; i.e. prior to adolescence, when the risk of
IM increases. Cost-effective deployment of a cheap, infection-preventing, vaccine with a lifelong duration of protection could thus likely involve targeting the early years. However, future vaccines may produce a shorter duration of immunity, potentially delaying infection and resulting in an increasing incidence of IM (and IMassociated cancers). This could be compounded by suboptimal vaccine coverage increasing the average age at infection [26] and consequently potentially increasing rates of IM - similarly to how sub-optimal coverage of the MMR vaccine led to an increase in congenital rubella syndrome in Greece [27, 28].

In such a scenario, targeted vaccine deployment to the social groups who acquire infection later (when the likelihood of IM is higher) might be considered, possibly with repeated dosing if required. Such targeting could be 


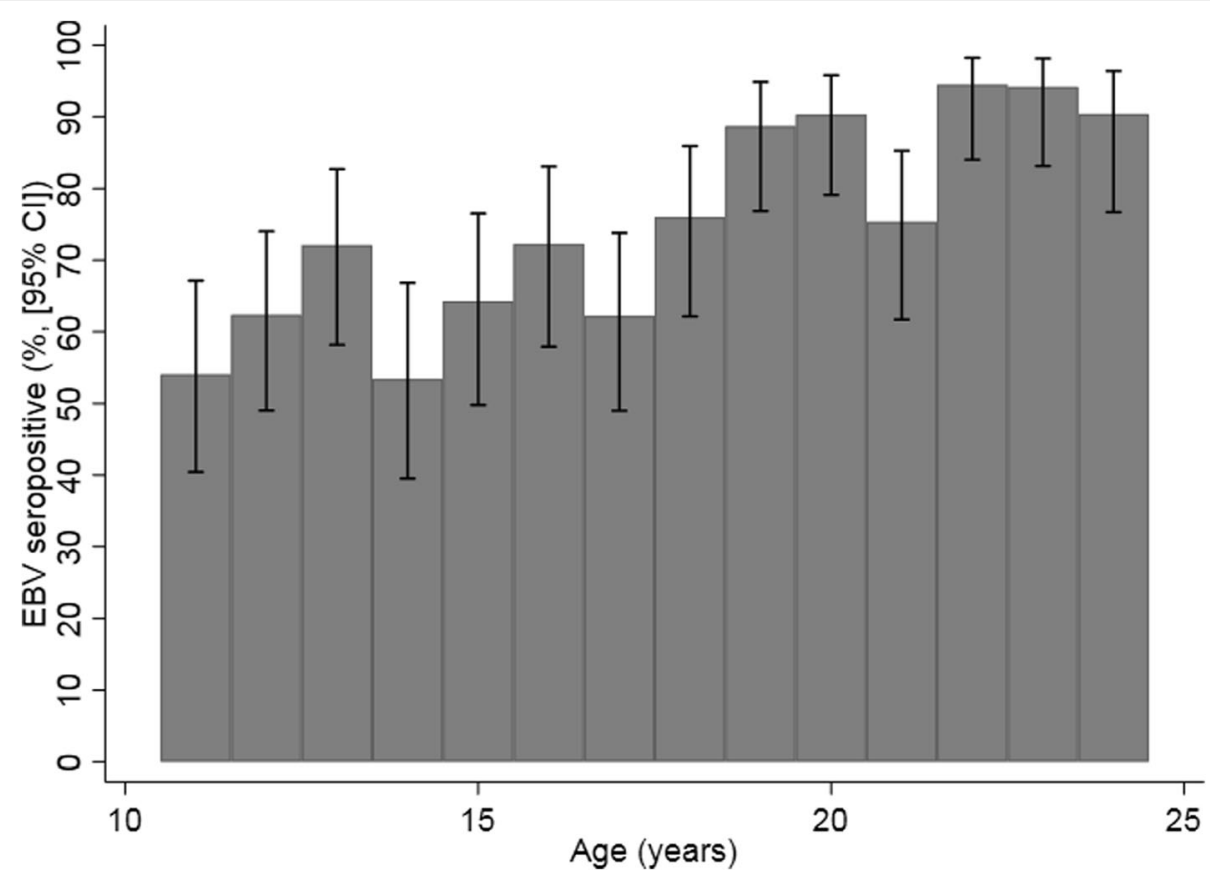

Fig. 2 Weighted seroprevalence of Epstein-Barr virus by age in England in 2002. Cl: confidence interval, EBV- Epstein Barr Virus

informed by the risk factors detected within this analysis, and data such as those presented here should be considered in conjunction with the characteristics of the vaccine available when determining what a vaccine policy should look like. If a vaccine was cheap and effective, then universal coverage would be appropriate. If the duration of protection was short, it may be prudent to give repeat doses of the vaccine to people who pick up the infection at the youngest age, which is linked to ethnicity and likely to socioeconomic status. The use of an expensive vaccine could be stratified on the basis of who is most likely to suffer EBV-related disease after infection, which we have studied separately [29].

The limitations of our work include the age of the data and the use of a cross-sectional study design, preventing determination of the temporality of the correlation between EBV and CMV infection. In our analysis, EBV seroprevalence was higher than CMV seroprevalence in all age groups, and both increased with age. We found that CMV was associated with EBV in univariable analyses, and in the adults-only model, but not in the whole-cohort multivariable model. As both EBV and CMV are associated with increasing age, particularly during adolescence, we would not expect an association between CMV and EBV to persist in the whole-cohort multivariable model. It is possible that as the association between age and EBV seroprevalence was less strong in the adults-only multivariable model (as EBV seroprevalence starts to saturate as people reach adulthood), there was enough of a residual effect that the association between EBV and CMV could be detected. Unfortunately, our sample size was not large enough to investigate the interactions between EBV, CMV and age in more detail. The association may result from shared genetic, immunological and/or sociodemographic risk factors, or one infection could increase susceptibility to the other. Longitudinal studies with serial testing are necessary to explore this association, and additional risk factors, in more detail.

We elected to measure IgG antibodies to the EBV VCA protein and whole CMV virus, as these antibodies are present in all infected individuals and persist for life. Although we did not test for IgM antibodies, and cannot exclude the possibility that some seronegative individuals may have been recently infected, we note that VCAspecific IgG and IgM antibodies usually appear contemporaneously [30] and therefore we would expect the number of such individuals in our study to be low.

\section{Conclusions}

Knowledge of the distribution of EBV infection among young population groups in England is critical for determining future vaccination policies, including the costeffectiveness of general versus selective approaches. Data such as those presented here should be used together with detailed information on vaccine characteristics, the implications of remaining EBV-uninfected for life, the ramifications of delayed infection, and the financial costs of IM and EBV-associated cancers to inform such policies. 
Table 2 Univariable and multivariable logistic regression models of factors associated with Epstein-Barr Virus seropositivity in England in 2002

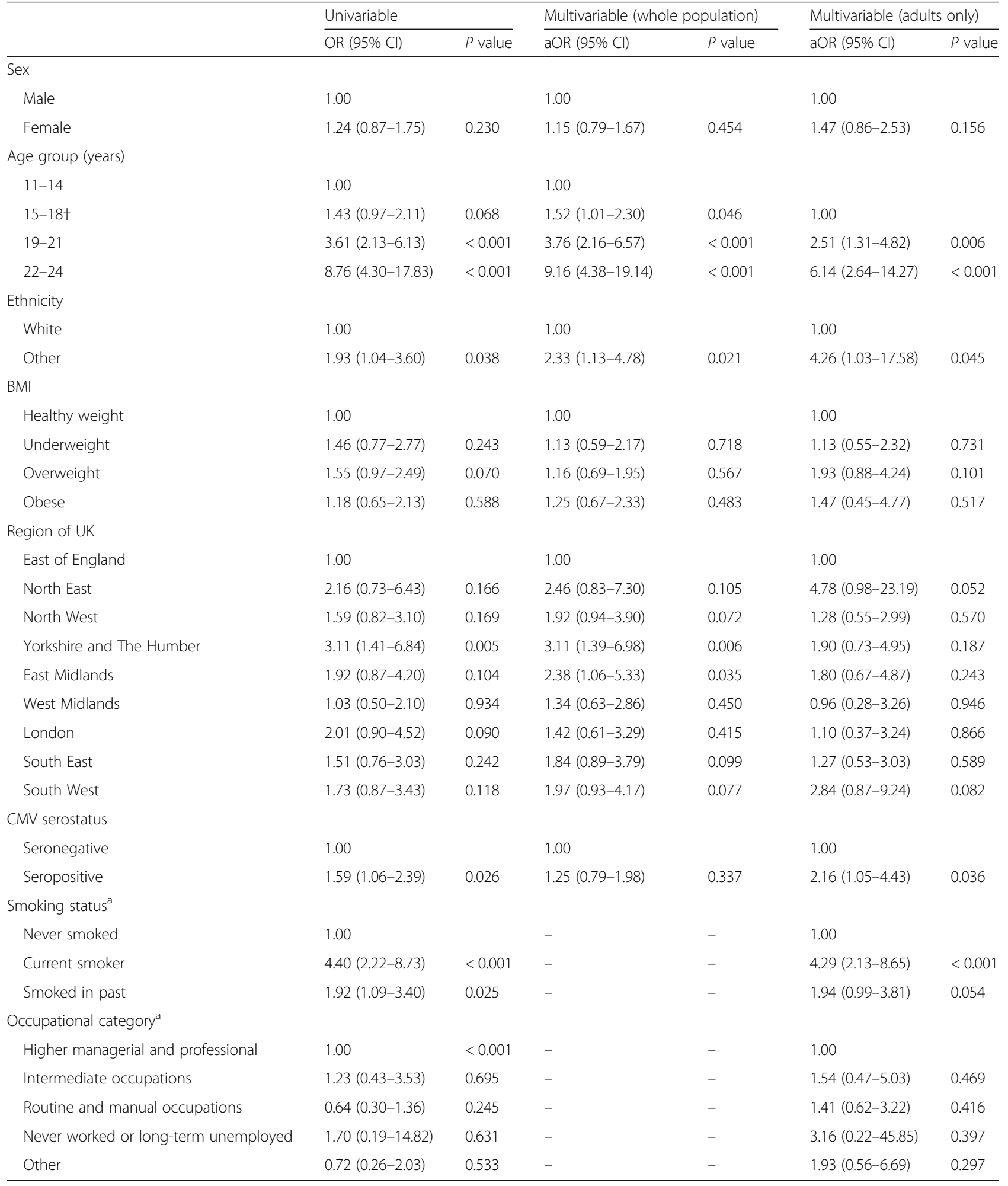

${ }^{a}$ Adults aged $\geq 16$ years only $(n=472)$. $+16-18$ years for 'adult-only' model. Odds ratios account for the weighting of the sample to be representative of the English population in 2002 with respect to age and sex. The 'whole population' multivariable model included age, sex, CMV serostatus, ethnicity, BMI and region of England. The 'adults only' multivariable model included all variables shown in the table. $a \mathrm{OR}$ adjusted odds ratio, $\mathrm{BMI}$ body mass index, $\mathrm{Cl}$ confidence interval, $C M V$ cytomegalovirus, $O R$ unadjusted odds ratio 


\section{Supplementary information}

Supplementary information accompanies this paper at https://doi.org/10. 1186/s12879-019-4578-y.

Additional file 1: Table S1. Distribution of study participants by age and gender. Table S2. ELISA assay performance.0020. Table S3. Sensitivity analysis of multivariable logistic regression models of factors associated with Epstein-Barr Virus seropositivity in England in 2002, excluding Cytomegalovirus serostatus as a risk factor. Table S4. Sensitivity analysis of multivariable logistic regression models of factors associated with Epstein-Barr Virus seropositivity in England in 2002, with indeterminate serology results reclassified as seronegative rather than seropositive.

\section{Abbreviations}

aOR: Adjusted odds ratio; BMI: Body mass index; Cl: Confidence interval; CMV: Cytomegalovirus; EBV: Epstein-Barr virus; ELISA: Enzyme-linked immunosorbent assay; HSE: Health Survey for England; IM: Infectious mononucleosis; NS-SEC: National statistics socio-economic classification; $\mathrm{RU}$ : Relative units; VCA: Virus capsid antigen

\section{Acknowledgements}

We thank our colleagues at UCL and NatCen Social Research, and the interviewers, research nurses and participants of the Health Survey for England, and Shaun Scholes for assistance weighting the HSE data for analysis.

\section{Authors' contributions}

HRS, JL and GT designed the study. OT and GT conducted the serologica testing. JRW conducted the data analysis and drafted the paper. JRW, CJ, $H R S, J L$ and GT interpreted the results. All authors critically revised the paper and approved the final version for publication.

\section{Funding}

This work was supported by the Wellcome Trust [204419]. The funding source has no role in the study design, collection, analysis or interpretation of the data, the writing of the paper or the decision to submit for publication. The corresponding author had full access to all data in the study and had final responsibility to submit the paper for publication.

\section{Availability of data and materials}

The data used in this study was under license from the Health Survey for England, and so are not publicly available, but can be requested from the HSE.

\section{Ethics approval and consent to participate}

This study was approved by the University College London Research Ethics Committee (5683/002). The HSE obtained informed written consent for blood samples to be collected and stored for future analyses. We were given permission to access the data by the Health Survey for England.

\section{Consent for publication}

Not applicable

\section{Competing interests}

GT reports personal fees from Genocea Biosciences, outside the submitted work. $\mathrm{CJ}$ is an Associate Editor at BMC Public Health. All other authors have no competing interests.

\footnotetext{
Author details

${ }^{1}$ Centre for Molecular Epidemiology and Translational Research, Institute for Global Health, University College London, London, UK. ${ }^{2}$ Institute of Immunology and Immunotherapy, College of Medical and Dental Sciences, University of Birmingham, Birmingham, UK. ${ }^{3}$ Current address: MRC Clinical Trials Unit, University College London, London, UK. ${ }^{4}$ National Institute for Health Research (NIHR) Health Protection Research Unit in Modelling Methodology, and Medical Research Council Centre for Outbreak Analysis and Public Health, Department of Infectious Disease Epidemiology, Imperial College London, London, UK. ${ }^{5}$ Usher Institute of Population Health Sciences and Informatics, University of Edinburgh, Edinburgh, UK.
}

Received: 20 June 2019 Accepted: 18 October 2019

Published online: 28 November 2019

\section{References}

1. Chang CM, et al. The extent of genetic diversity of Epstein-Barr virus and its geographic and disease patterns: a need for reappraisal. Virus Res. 2009; 143(2):209-21.

2. Houldcroft CJ, Kellam P. Host genetics of Epstein-Barr virus infection, latency and disease. Rev Med Virol. 2015;25(2):71-84.

3. Evans AS. Infectious mononucleosis and related syndromes. Am J Med Sci. 1978;276(3):325-39.

4. Macsween $\mathrm{KF}$, et al. Infectious mononucleosis in university students in the United Kingdom: evaluation of the clinical features and consequences of the disease. Clin Infect Dis. 2010;50(5):699-706.

5. Visser $\mathrm{E}$, et al. The epidemiology of infectious mononucleosis in northern Scotland: a decreasing incidence and winter peak. BMC Infect Dis. 2014;14:151.

6. Parkin DM. The global health burden of infection-associated cancers in the year 2002. Int J Cancer. 2006;118(12):3030-44.

7. Sokal EM, et al. Recombinant gp350 vaccine for infectious mononucleosis: a phase 2, randomized, double-blind, placebo-controlled trial to evaluate the safety, immunogenicity, and efficacy of an Epstein-Barr virus vaccine in healthy young adults. J Infect Dis. 2007;196(12):1749-53.

8. Kanekiyo $\mathrm{M}$, et al. Rational Design of an Epstein-Barr Virus Vaccine Targeting the receptor-binding site. Cell. 2015;162(5):1090-100.

9. Hjalgrim H, Friborg J, Melbye M. In: Arvin A, et al., editors. The epidemiology of EBV and its association with malignant disease, in Human herpesviruses: Biology, Therapy and Immunoprophylaxis. Cambridge: Cambridge University Press; 2007.

10. Piriou $\mathrm{E}$, et al. Early age at time of primary Epstein-Barr virus infection results in poorly controlled viral infection in infants from Western Kenya: clues to the etiology of endemic Burkitt lymphoma. J Infect Dis. 2012;205(6):906-13.

11. Slyker JA, et al. Clinical and virologic manifestations of primary Epstein-Barr virus (EBV) infection in Kenyan infants born to HIV-infected women. J Infect Dis. 2013;207(12):1798-806.

12. Winter JR, et al. Predictors of Epstein-Barr virus serostatus and implications for vaccine policy: a systematic review of the literature. Under review.

13. Gosce $L$, et al. Modelling the dynamics of EBV transmission to inform a vaccine target product profile and future vaccination strategy. Sci Rep. 2019; 9(1):9290.

14. Pembrey L, et al. Seroprevalence of cytomegalovirus, Epstein Barr virus and varicella zoster virus among pregnant women in Bradford: a cohort study. PLOS ONE. 2013;8:e81881.

15. Kessell I, et al. Epstein-Barr virus infection in children entering a paediatric unit. J Inf Secur. 1980;2(3):269-74.

16. Crawford DH, et al. A cohort study among university students: identification of risk factors for Epstein-Barr virus seroconversion and infectious mononucleosis. Clin Infect Dis. 2006:43(3):276-82.

17. Deverill, C., et al., Health Survey for England 2002: The Health of Children and Young People. Methodology \& Documentation, ed. K. Sproston and P. Primatesta. 2002: The Stationery Office.

18. Health \& Social Care Information Centre, The Health Survey for England Bloodbank Project: Requests for extraction and analysis of HSE stored blood samples. 2013.

19. Office for National Statistics. Dataset: Population Estimates for UK, England and Wales, Scotland and Northern Ireland. 2018 16/01/2018]; Available from: https://www.ons.gov.uk/peoplepopulationandcommunity/ populationandmigration/populationestimates/datasets/ populationestimatesforukenglandandwalesscotlandandnorthernireland.

20. Office for National Statistics. 2011 Census: boundary data (United Kingdom) [data collection]. 2011 16/01/2018]; Available from: http://census. ukdataservice.ac.uk/get-data/boundary-data.aspx.

21. Office for National Statistics. The National Statistics Socio-economic classification (NS-SEC). 2019 06/10/2019].

22. Cohen Jl, et al. Epstein-Barr virus: an important vaccine target for cancer prevention. Sci Transl Med. 2011;3(107):107fs7.

23. Simanek AM, et al. Seropositivity to cytomegalovirus, inflammation, all-cause and cardiovascular disease-related mortality in the United States. PLoS One. 2011;6(2):e16103.

24. Levine $\mathrm{H}$, et al. Seroepidemiology of Epstein-Barr virus and cytomegalovirus among Israeli male young adults. Ann Epidemiol. 2012;22:783-8. 
25. Lazda VA. Evaluation of Epstein-Barr virus (EBV) antibody screening of organ donors for allocation of organs to EBV serostatus matched recipients. Transplant Proc. 2006;38(10):3404-5.

26. Vynnycky, E. and R.G. White, An introduction to infectious disease modelling. 2010: Oxford University Press.

27. Panagiotopoulos T, Antoniadou I, Valassi-Adam E. Increase in congenital rubella occurrence after immunisation in Greece: retrospective survey and systematic review. BMJ. 1999;319(7223):1462-7.

28. Panagiotopoulos T, Georgakopoulou T. Epidemiology of rubella and congenital rubella syndrome in Greece, 1994-2003. Euro Surveill. 2004; 9(4):17-9.

29. Bakkalci $D$, et al. Risk factors for Epstein-Barr virus-associated cancers: a systematic review, critical appraisal, and mapping of the epidemiological evidence. Under review.

30. De Paschale M, Clerici P. Serological diagnosis of Epstein-Barr virus infection: problems and solutions. World J Virol. 2012;1(1):31-43.

\section{Publisher's Note}

Springer Nature remains neutral with regard to jurisdictional claims in published maps and institutional affiliations.

Ready to submit your research? Choose BMC and benefit from:

- fast, convenient online submission

- thorough peer review by experienced researchers in your field

- rapid publication on acceptance

- support for research data, including large and complex data types

- gold Open Access which fosters wider collaboration and increased citations

- maximum visibility for your research: over $100 \mathrm{M}$ website views per year

At BMC, research is always in progress.

Learn more biomedcentral.com/submissions 